\title{
Nonlinear beam cleanup in Yb-doped GRIN multimode fiber taper
}

\author{
A. Niang ${ }^{1}$, D. Modotto ${ }^{1}$, A. Tonello ${ }^{2}$, F. Mangini ${ }^{1}$, U. Minoni ${ }^{1}$, M. Zitelli ${ }^{3}$, M. Fabert ${ }^{2}$, \\ M.A. Jima ${ }^{1,2}$, O.N. Egorova $^{4}$, A.E. Levchenko ${ }^{5}$, S.L. Semjonov ${ }^{5}$, D.S. Lipatov ${ }^{6}$, \\ S. Babin ${ }^{7,8}$, V. Couderc ${ }^{2}$, and S. Wabnitz ${ }^{3,7}$ \\ ${ }^{1}$ Dipartimento di Ingegneria dell'Informazione, Università di Brescia, via Branze 38, 25123, Brescia, Italy \\ ${ }^{2}$ Université de Limoges, XLIM, UMR CNRS 7252, 123 Avenue A. Thomas, 87060 Limoges, France \\ ${ }^{3}$ Dipartimento di Ingegneria dell'Informazione, Elettronica e Telecomunicazioni, Sapienza University of Rome, \\ Via Eudossiana 18, 00184 Rome, Italy \\ ${ }^{4}$ Prokhorov General Physics Institute of the Russian Academy of Sciences, 38 Vavilov Street, 119991 Moscow, \\ Russia \\ ${ }^{5}$ Fiber Optics Research Center of the Russian Academy of Sciences, 38 Vavilov Street, 119333 Moscow, Russia \\ ${ }^{6}$ Devyatykh Institute of Chemistry of High-Purity Substances of the Russian Academy of Sciences, 49 Tropinin \\ Street, 603950 Nizhny Novgorod, Russia \\ ${ }^{7}$ Novosibirsk State University, Pirogova 1, Novosibirsk 630090, Russia \\ ${ }^{8}$ Institute of Automation and Electrometry of the Russian Academy of Sciences, Koptyuga 1, Novosibirsk 630090, \\ Russia \\ alioune.niang@unibs.it
}

\begin{abstract}
We demonstrate beam self-cleaning in a tapered Ytterbium-doped gradedindex multimode fiber in both active and passive configurations, without accompanying self-phase modulation induced spectral broadening or frequency conversion. (c) 2020 The Author(s)
\end{abstract}

Nonlinear effects in multimode fibers (MMFs) are extensively studied in recent years. Their specific nonlinear properties include the reshaping a highly irregular or speckled transverse spatial beam pattern at the fiber output, by means of either Raman and Brillouin scattering, or by the Kerr effect. All of these nonlinear processes lead to spatial beam cleaning, thus generating high quality output beams. On the other hand, the available gain in fiber lasers, provided by active rare-earth doping [1,2], is limited by the presence of Raman and Brillouin scattering, and transverse mode instabilities (TMI) [3,4]. Using a tapered Yb-doped (YD) MMFs, with a varying fiber core size along its axis, is a solution to mitigate TMI, hence to obtain high output quality beams by filtering high-order modes [5].

Previously, we experimentally demonstrated Kerr-beam self-cleaning (KBSC) in a $10 \mathrm{~m}$ long tapered, YD graded-index (GRIN) MMF when pulses were launched from the wider $(120 \mu \mathrm{m})$ into the smaller $(40 \mu \mathrm{m})$ core [6]. We observed self-cleaning without significant frequency conversion in the passive regime (without the pump laser diode). On the other hand, the presence of gain from the pump led to a beam cleanup which was accompanied by ultrawideband frequency conversion and supercontinuum generation, extending from $520 \mathrm{~nm}$ to $2600 \mathrm{~nm}$ [6]. It should be noted that frequency conversions should be avoided in high power fiber lasers. For this reason, the laser pulse should propagate from the smaller to the wider diameter of tapered-Yb doped fibers, which could decrease the fiber nonlinearity as the pulse get amplified.

In this work, we experimentally demonstrate spatial KBSC in a tapered YD MMF, where the laser pulse propagates from the smallest to the widest core. The experimental setup is described [5]. Briefly, the injected laser pulses at $1064 \mathrm{~nm}$, propagating from the small $(47 \mu \mathrm{m})$ to the large $(96 \mu \mathrm{m})$ diameter of a $5 \mathrm{~m}$ tapered YD GRIN MMF. A pump CW diode laser at $940 \mathrm{~nm}$ was placed in a counter directional configuration.

In a passive configuration, the output spatial beam pattern was speckled for low input power laser pulses. We gradually increased input peak power $\left(P_{\text {in }}\right)$ from $280 \mathrm{~W}$ up to $51 \mathrm{~kW}$ (corresponding to the damage threshold of the fiber end face). As shown in the insets of Fig. 1a, for $P_{i n}>20 \mathrm{~kW}$ the speckled output intensity pattern self-organized into a bell-shaped beam, a clear demonstration of KBSC. The observed self cleaning can be explained in terms of the nonlinear nonreciprocity of the mode coupling process. From beam quality parameter $M^{2}$ measurements, see Fig. 1a, we confirmed that the output beam quality improves by increasing the input peak power. $M^{2}$ drops from $M^{2}=12$ to $M^{2}=4$ by increasing $P_{\text {in }}$ up to $20 \mathrm{~kW}$, and then it stabilizes around $M^{2}=3$ by further increasing the input power. The reduction of $M^{2}$ is accompanied by a strong environmental stability of the self-cleaned beam. Self-cleaning occurs in the absence of any spectral broadening, and frequency conversion processes.

In the active configuration, we turned on the CW diode laser pump, and placed a bandpass filter centered at $1064 \mathrm{~nm}$ with $10 \mathrm{~nm}$ bandwidth in front of camera, to block residual light from the pump diode laser, as well as amplified spontaneous emission (ASE) noise. The input peak power was fixed at $10 \mathrm{~kW}$, below the self-cleaning 


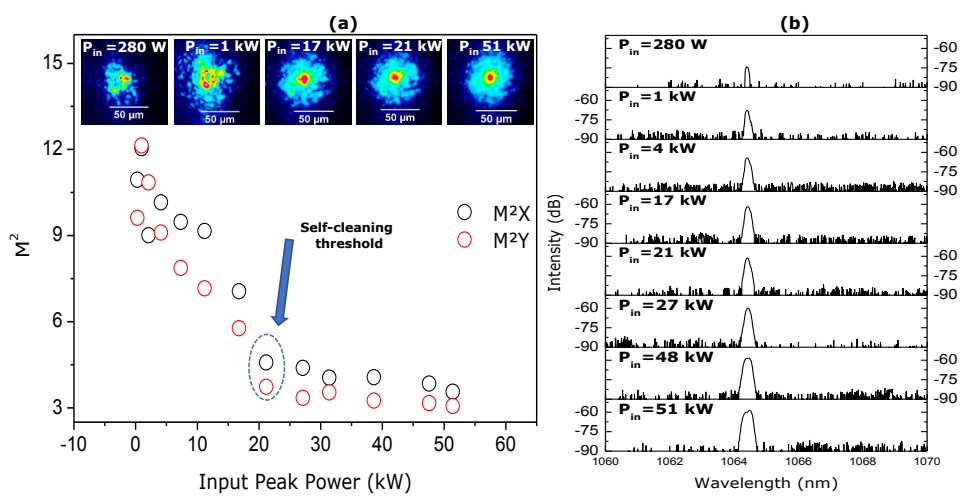

Fig. 1. Passive configuration: (a) Beam quality $M^{2}$ parameter vs. input peak power; insets show near-field spatial intensity distributions, (b) spectra as function of input peak power.
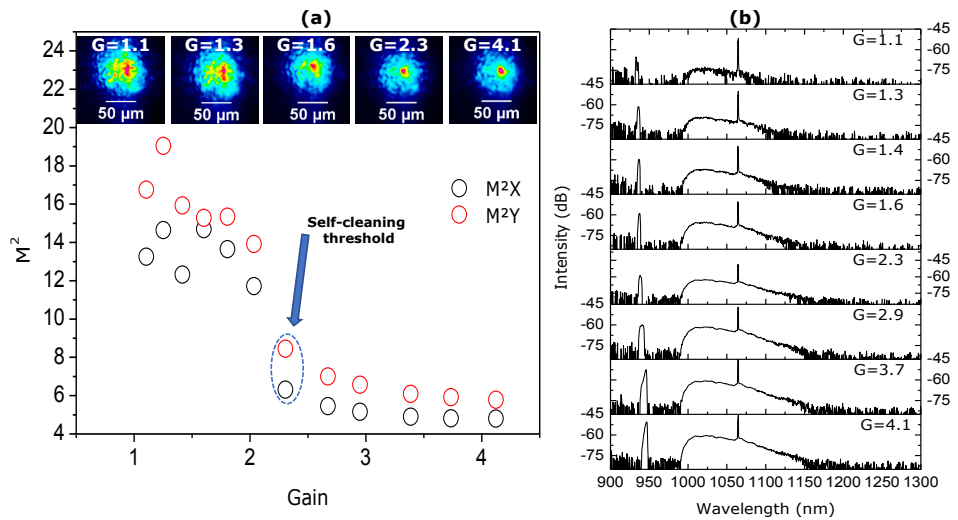

Fig. 2. Active configuration: (a) Beam quality $M^{2}$ parameter vs. input peak power; insets show nearfield spatial intensity distributions, (b) spectra as function of input peak power.

threshold in the passive configuration. The presence of gain was sufficient to introduce beam self-cleaning. As shown in the insets of Fig. 2a, the output beam pattern was speckled in the absence of gain. By increasing the gain (defined as the ratio between output power with and without pump), a self-organization of the speckled beam led to self-cleaning for a gain $G>1.6$. For $M^{2}$ measurements, a $3 \mathrm{~nm}$ bandwidth filter at $1064 \mathrm{~nm}$ was used to further reduce the influence of output ASE, appearing as a low-power background. As can be seen in Fig. 2a, the output beam quality parameter $M^{2}$ sharply dropped from $M^{2}=15$ for $\mathrm{G}=1.1$ down to $M^{2}=5$ for $\mathrm{G}=4$. 1 , when the amplified laser beam was injected from the smaller side of the fiber. Even in the active configuration, self-cleaning was not accompanied by any spectral broadening nor frequency conversion, see Fig. 2 b.

To conclude, we experimentally demonstrated Kerr beam self-cleaning in a tapered YD MMF operating in either active or passive configurations. Due to self-cleaning, the speckled output pattern self-organized into a bell-shaped output beam pattern. Obtaining self-cleaning with with improved beam quality factor and no spectral broadening or frequency conversion provides a remarkable nonlinear multimode propagation property of tapered multimode fibers. Kerr self-cleaning combined with core size widening along the propagation direction lead to a progressive trapping of the beam towards the fundamental mode. We envisage that nonlinear multimode tapered amplifiers could be a building block for a future generation of high beam quality, and high-power multimode fiber lasers.

We acknowledge the financial support from the European Research Council (grant No. 740355) and the Russian Ministry of Science and Education (grant No. 14.Y26.31.0017).

\section{References}

1. C. Jauregui, et al., Nat. Photon 7, 861-867 (2013).

2. J. Nilsson, et al., Science 332, 921-922 (2011).

3. T. Eidam, et al., Opt. Express 19, 13218-13224 (2011).

4. C. Jauregui, et al., Opt. Express 19, 3258-3271 (2011).

5. A. Niang, et al., IEEE Photonics J., 10.1109/JPHOT.2020.2979938 (2020).

6. A. Niang, et al., Opt. Express, 27, 24018-24028 (2019). 